PREPARED FOR THE U.S. DEPARTMENT OF ENERGY, UNDER CONTRACT DE-AC02-76CH03073

PPPL-3914

PPPL-3914

UC-70

Simulation of Optical and Synthetic Imaging using Microwave Reflectometry

by

G.J. Kramer, R. Nazikian, and E. Valeo

January 2004

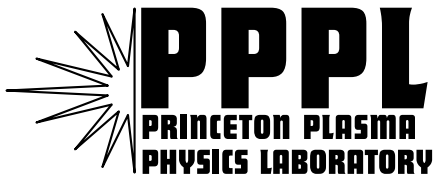

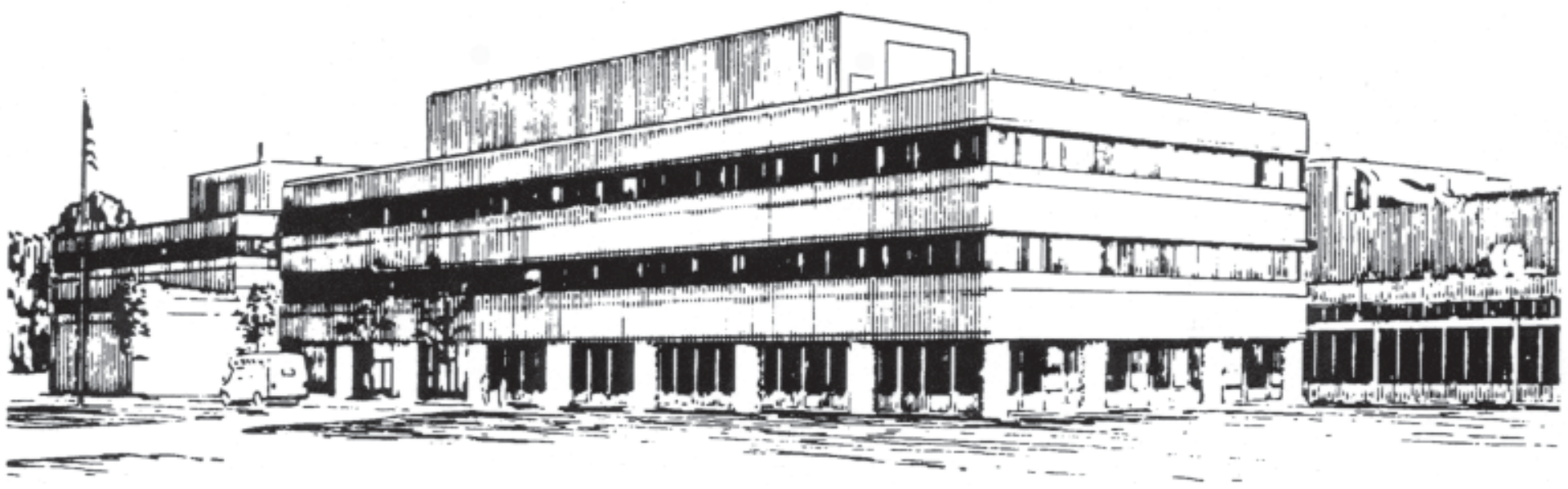

PRINCETON PLASMA PHYSICS LABORATORY PRINCETON UNIVERSITY, PRINCETON, NEW JERSEY 


\section{PPPL Reports Disclaimer}

This report was prepared as an account of work sponsored by an agency of the United States Government. Neither the United States Government nor any agency thereof, nor any of their employees, makes any warranty, express or implied, or assumes any legal liability or responsibility for the accuracy, completeness, or usefulness of any information, apparatus, product, or process disclosed, or represents that its use would not infringe privately owned rights. Reference herein to any specific commercial product, process, or service by trade name, trademark, manufacturer, or otherwise, does not necessarily constitute or imply its endorsement, recommendation, or favoring by the United States Government or any agency thereof. The views and opinions of authors expressed herein do not necessarily state or reflect those of the United States Government or any agency thereof.

\section{Availability}

This report is posted on the U.S. Department of Energy's Princeton Plasma Physics Laboratory Publications and Reports web site in Fiscal Year 2004. The home page for PPPL Reports and Publications is: http://www.pppl.gov/pub_report/

DOE and DOE Contractors can obtain copies of this report from:

U.S. Department of Energy

Office of Scientific and Technical Information

DOE Technical Information Services (DTIS)

P.O. Box 62

Oak Ridge, TN 37831

Telephone: (865) 576-8401

Fax: (865) 576-5728

Email: reports@adonis.osti.gov

This report is available to the general public from:

National Technical Information Service

U.S. Department of Commerce

5285 Port Royal Road

Springfield, VA 22161

Telephone: $1-800-553-6847$ or

(703) $605-6000$

Fax: (703) 321-8547

Internet: http://www.ntis.gov/ordering.htm 


\title{
Simulation of optical and synthetic imaging using microwave reflectometry
}

\author{
G J Kramer, R Nazikian and E Valeo \\ Princeton Plasma Physics Laboratory Princeton NJ 08543-0451 USA \\ E-mail: gkramer@pppl.gov
}

\begin{abstract}
D full-wave time-dependent simulations in full plasma geometry are presented which show that conventional reflectometry (without a lens) can be used to synthetically image density fluctuations in fusion plasmas under conditions where the parallel correlation length greatly exceeds the poloidal correlation length of the turbulence. The advantage of synthetic imaging is that the image can be produced without the need for a large lens of high optical quality, and each frequency that is launched can be independently imaged. A particularly simple arrangement, consisting of a single receiver located at the midpoint of a microwave beam propagating along the plasma midplane is shown to suffice for imaging purposes. However, as the ratio of the parallel to poloidal correlation length decreases, a poloidal array of receivers needs to be used to synthesize the image with high accuracy. Simulations using DIIID relevant parameters show the similarity of synthetic and optical imaging in present day experiments.
\end{abstract}

Submitted to: Plasma Phys. Control. Fusion 


\section{1. introduction}

For many years it has been argued that reflectometry can be used to image poloidal density fluctuations in fusion plasmas $[1,2]$. The essence of this claim is based on the validity of geometric optics near the reflecting layer. By this we mean that if the density fluctuations are sufficiently smooth and sufficiently weak that they predominantly change the phase of the wave front without affecting its amplitude, then these phase modulations can in principle be imaged onto a detector plane by using a lens located outside the plasma. The quantitative interpretation of the measured signals in terms of density fluctuations at the reflecting layer is straight forward provided these fluctuations primarily affect the phase of the reflected waves. However, it was also shown [3] that imaging can be performed even in the absence of a lens provided the fluctuations behave as frozen structures that drifted poloidally across the detector array. This result, if generally true, is of considerable importance as it would mean that the expense and technical difficulties of constructing a lens based imaging system can be avoided. Till recently there has not been the capability to model different imaging methods in order to assess their relative merits in realistic geometries. In this paper we show that synthetic imaging is practical for single frequencies, and potentially more practical for multiple frequency beams in present day and future plasma experiments. The basis of this evaluation is the simulation of many thousands of reflectometer measurements in full plasma geometry using a highly efficient full wave algorithm [4].

Full wave simulations of reflectometry in a slab geometry with incident plane waves has been used in the past to demonstrate the possibility of imaging density fluctuations near a cutoff layer $[5,6]$. However, when dealing with microwaves in the real world one has to deal with nontrivial matters such as the curvature of the reflecting layer, the up/down asymmetry in the plasma, the finite extent and divergence of the incoming beam, the finite aperture of the receiver and not least, diffraction effects from limiting apertures such as lenses. These issues all have an impact on the efficacy of imaging reflectometry in fusion plasmas. For example, the importance of plasma curvature and beam divergence was clearly identified when a realistic simulation was performed for Alcator C-mod conditions, leading to the discovery of the phenomenon of super resolution as it applies to reflectometry [7]. By super resolution it is meant that a standard microwave receiver can have a greatly increased wavenumber resolution than its apparent aperture suggests simply by the divergence of the beam from the curved cutoff layer. Such nontrivial effects must be taken into account when judging the applicability of optical or synthetic imaging in fusion plasmas.

Although optical imaging has been vigorously pursued in recent years, [6, 8, 9, 10] synthetic imaging has not received the same degree of attention despite its obvious advantages and relative simplicity. In the fact that the first demonstration of imaging using reflectometry was based on a synthetic imaging algorithm applied to conventional reflectometer data taken on TFTR [3]. Here the method of synthetic imaging is based on the application of a numerical back projection algorithm. By the assumption of frozen 
poloidal flow of density fluctuations, the imaging scheme becomes particularly simple, requiring only a single detector. The key element in this method is the requirement of a high degree of poloidal coherence in the detected signal [11].

In this paper we discuss the relative merit and range of applicability of optical (with lens) and synthetic (without lens) imaging using time dependent simulations of 2-D reflectometry in real plasma geometry. The full wave simulation code used to model real and synthetic imaging allows the generation of time series data formally equivalent to the signals detected in a real reflectometer experiment, and the results of the simulations are investigated using time series analysis tools that are also used in actual experiments, so that a robust determination can be performed of the possibility of imaging under conditions that closely mimic actual experiments. An important result from this work is that in rapidly rotating plasmas (such as in JET, DIII-D, and NSTX), the possibility of synthetic imaging may be pursued as a low cost method for measuring the poloidal structure of density fluctuations in the plasma. In addition, the method can be used to determine if a more sophisticated synthetic imaging system using a poloidal array of receivers is justifiable.

In section 2 details are presented on the full wave analysis code used to generate time series data. In section 3.1 the imaging properties of an optical reflectometer system with finite aperture effects and full plasma geometry are presented. Section 3.2 is devoted to synthetic imaging, based on single and multichannel approaches. In section 4 a comparison between synthetic and optical imaging is made under DIII-D relevant conditions and the limitations of the methods are presented. In section, 5 concluding remarks and future directions for reflectometer research are discussed.

\section{Time Dependent 2-D Full Wave Simulations}

For our study of the imaging properties of reflectometer systems, we have used the 2-D Full Wave Reflectometer code FWR2D [4] to simulate the electrical field scattered in the poloidal direction from random density fluctuations. FWR2D solves the time dependent scalar wave equation for the electrical Field $E(\mathbf{x}, t)$,

$$
\frac{\partial^{2} E}{\partial x^{2}}+\frac{\partial^{2} E}{\partial y^{2}}+k_{0}^{2} \epsilon E=2 i \omega \frac{\partial E}{\partial t}
$$

where $x$ is the radial direction on the plasma midplane, $y$ is the vertical (poloidal) direction, $k_{0}=\omega / c$, with $\omega$ the wave frequency and $c$ the speed of light in vacuum, and $\epsilon(\mathbf{x})$ the electric permittivity of the medium where $\epsilon=0$ at the reflecting layer and $\epsilon=1$ in vacuum. The computational domain is divided into three regions: the vacuum region, the paraxial region, and the full wave region. The waves are propagated in the vacuum region between the transmitter/detector plane and the plasma edge with the Fresnel-Huygens formula. From the plasma edge to the vicinity of the reflection layer a paraxial approximation is made to speed up the computation. We have verified that the paraxial solution is accurate by comparing it to the full wave solution in the same 
region. In the region around the reflection layer the full wave equation is solved. In the paraxial and full wave region the full 2-D plasma geometry is taken into account in the computations. (Further details on the calculations are given in [4]).

For the simulations in this paper we have used a DIII-D equilibrium with the following parameters: toroidal magnetic field $B=2.1 \mathrm{~T}$, plasma current $I_{p}=0.98 \mathrm{MA}$, major radius $R_{0}=1.66 \mathrm{~m}$, minor radius $a=0.64 \mathrm{~m}$, ellipticity 1.12 , triangularity 0.21 , and central plasma density $1.6 \times 10^{19} \mathrm{~m}^{-3}$. A microwave beam of $55 \mathrm{GHz}$ launched in the Extraordinary mode $(\mathbf{E} \perp \mathbf{B})$ propagates in from the low field side as shown in figure 1a. The antenna was positioned $1.5 \mathrm{~m}$ from the plasma edge and the reflection layer was located $10 \mathrm{~cm}$ inside the plasma.

In the absence of random variations in the plasma density, the wave equation need only be solved once for each geometry and wave frequency. However, in order to simulate turbulence with good statistical properties, many independent solutions to the wave equation are required. A time dependent random density field is constructed, comprising of 10000 time slices, with each time slice representing a two dimensional random density distribution (in $x, y$ ) with the following spectral properties:

$$
\frac{1}{n^{2}}\left\langle\tilde{n}_{1} \tilde{n}_{2}\right\rangle=\left(\frac{\tilde{n}}{n}\right)^{2} \exp \left(-\left(\frac{\Delta t}{\tau}\right)^{2}\right) \exp \left(-\left(\frac{(\mathbf{x}+\mathbf{v} t) \cdot \Delta \mathbf{k}}{2}\right)^{2}\right) \cos (\mathbf{x} \cdot \mathbf{k})
$$

with $\tilde{n} / n$ the density fluctuation level, $\tau$ the density decorrelation time, $\mathbf{v}$ the poloidal velocity of the turbulence, $\mathbf{k}$ the mean value of the fluctuation wave number, $\Delta \mathbf{k}$ its spread, and $\mathbf{x}=\left(x_{1}-x_{2}, y_{1}-y_{2}\right)$ with $x_{1}-x_{2}$ the radial and $y_{1}-y_{2}$ the poloidal

displacement. Unless otherwise stated, we have used the following parameters in our simulations: $\tilde{n} / n=0.1 \%, \tau=600 \mu \mathrm{s}, \mathbf{v}=\left(0.0,2.5 \times 10^{5}\right) \mathrm{cm} / \mathrm{s}, \mathbf{k}=(0,0) \mathrm{cm}^{-1}$, $\Delta \mathbf{k}=(\mathbf{1 . 0}, \mathbf{1 . 0}) \mathrm{cm}^{-1}$, and $\Delta t=1 \mu \mathrm{s}$.

The ensemble number is defined as the ratio of the reflectometer record length divided by the autocorrelation time of the electric field at the point of measurement. According to this criterion, the number of independent reflectometer realizations at the detector plane is typically 1600, allowing accurate statistical properties of the scattered field to be deduced.

\section{Full Wave Simulation of Imaging Reflectometry}

\subsection{Optical Imaging}

In a conventional reflectometer system a narrow beam of microwaves is broadcast into the plasma and the reflected waves are detected with the transmitter antenna or with a receiver antenna located close to the transmitter. Because of the plasma curvature and the curvature of the wave front the scattered power is spread out widely at the antenna plane (figure $1 \mathrm{a}$ ).

In an imaging reflectometer system a large aperture lens is used to focus the reflected waves and to form an image at the image plane (figure $1 \mathrm{~b}$ ) $[6,9]$. When the fluctuations 


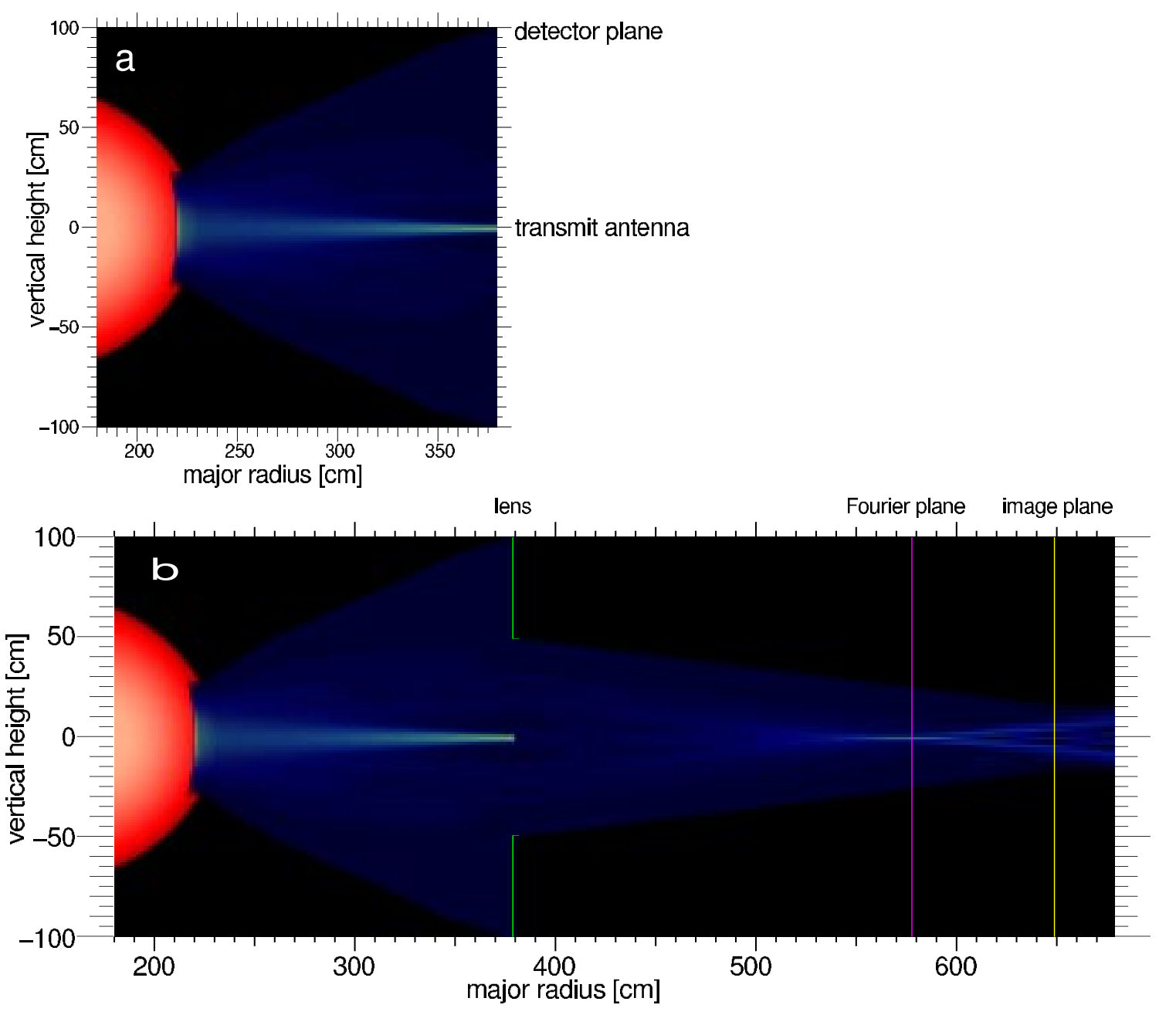

Figure 1. (a) The microwave intensity, $|E|^{2}$, for a conventional reflectometer system and (b) for an imaging system. In both cases the transmitter is located at the midplane at $3.8 \mathrm{~m}$ from the torus center. For the imaging system a lens with an aperture of $1 \mathrm{~m}$ and a focal length of $1 \mathrm{~m}$ was placed at the detector plane. The Focal plane of the lens (hereby termed the Fourier plane) is formed at $5.8 \mathrm{~m}$ (purple line) and the image plane is at $6.4 \mathrm{~m}$ (yellow line).

are weak the Fourier plane is characterized by a maximum in the electric field along the optical axis (figure 2 a). This plane corresponds to the location where the unscattered waves are most strongly convergent. When the density fluctuations at the reflection layer increase, the focal point at the Fourier plane broadens and the intensity decreases as is shown in figure $2 \mathrm{~b}-\mathrm{d}$ for fluctuation levels of $0.1,0.5$, and $1.5 \%$.

At the Fourier plane on the optical axis the amplitude fluctuations, calculated as $\left\langle\tilde{A}^{2}\right\rangle / A^{2}=\langle|E|-\langle|E|\rangle\rangle^{2} /\langle|E|\rangle^{2}$ (where $\langle\ldots\rangle$ stands for ensemble averaging), reaches a minimum (figure $2 \mathrm{e}$ ). This minimum disappears quickly when the density fluctuation level at the cut off increases. A second minimum in the amplitude fluctuations is found at the image plane (figure $2 \mathrm{f}-\mathrm{h}$ ). This means that all the information on the density fluctuations at the cut off layer is contained in the phase fluctuations at the image plane 

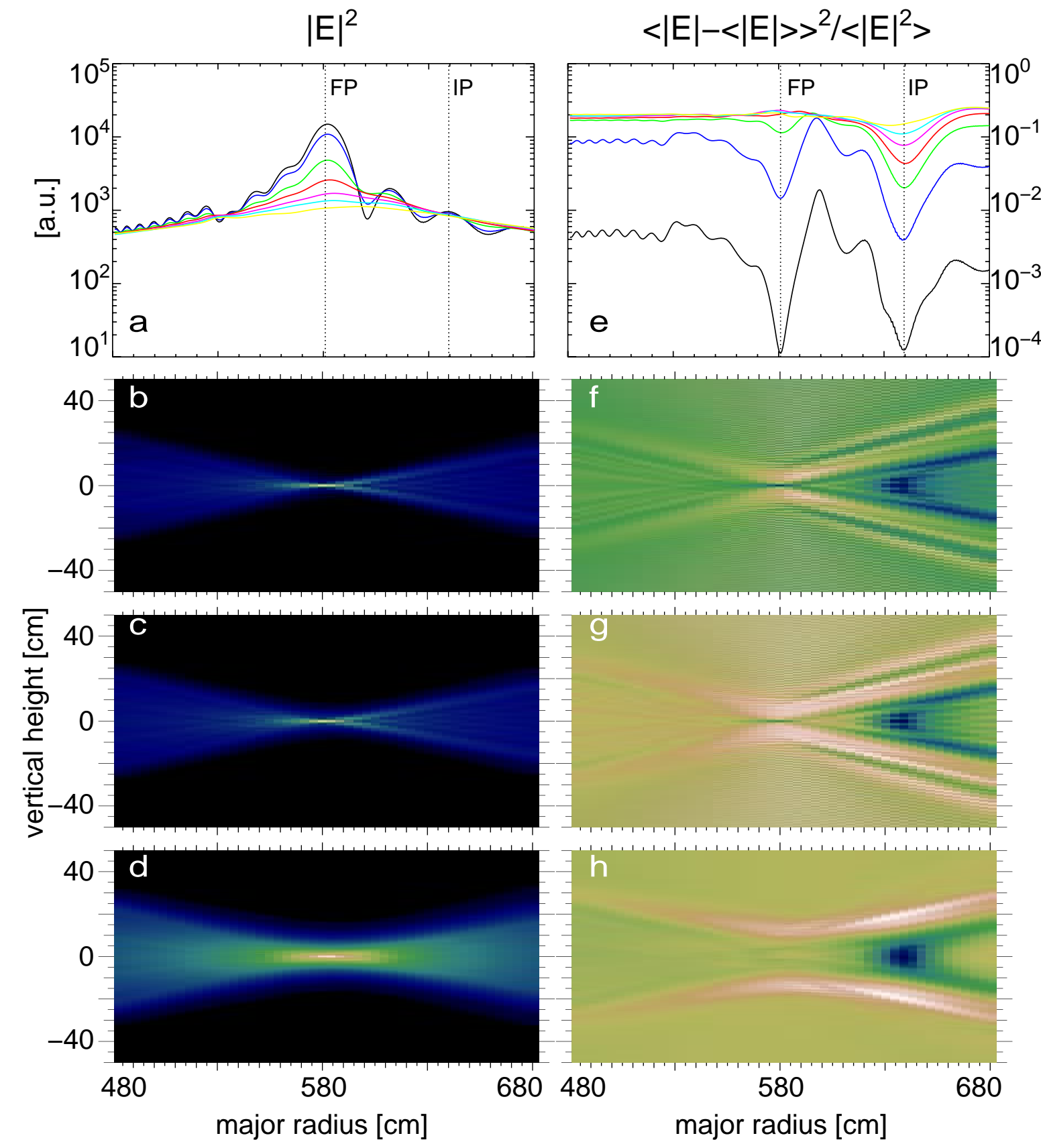

Figure 2. (a) The intensity, $\left\langle|E|^{2}\right\rangle$, of the electrical field along the optical axis for density fluctuation levels of $0.1 \%$ (black), $0.5 \%$ (blue), $1.0 \%$ (green), $1.5 \%$ (red), $2.0 \%$ (magenta), $2.5 \%$ (cyan), and $3.0 \%$ (yellow). The intensity of $\left\langle|E|^{2}\right\rangle$ as a function of major radius and vertical height is shown for $\tilde{n} / n=0.1 \%$ (b), $0.5 \%$ (c), and $1.5 \%$ (d). The intensity increases from black via blue, green, yellow, and pink to white. (e) The relative amplitude fluctuation level along the optical axis (same color scheme as in (a)). The amplitude fluctuation level as a function of major radius and vertical height for $0.1 \%$ (f), $0.5 \%$ (g), and $1.5 \%$ (h). The fluctuation level increase from black via blue, green, yellow, and pink to white. In (a) and (e) the Fourier plane (FP) and image plane (IM) are indicated with dotted lines. 


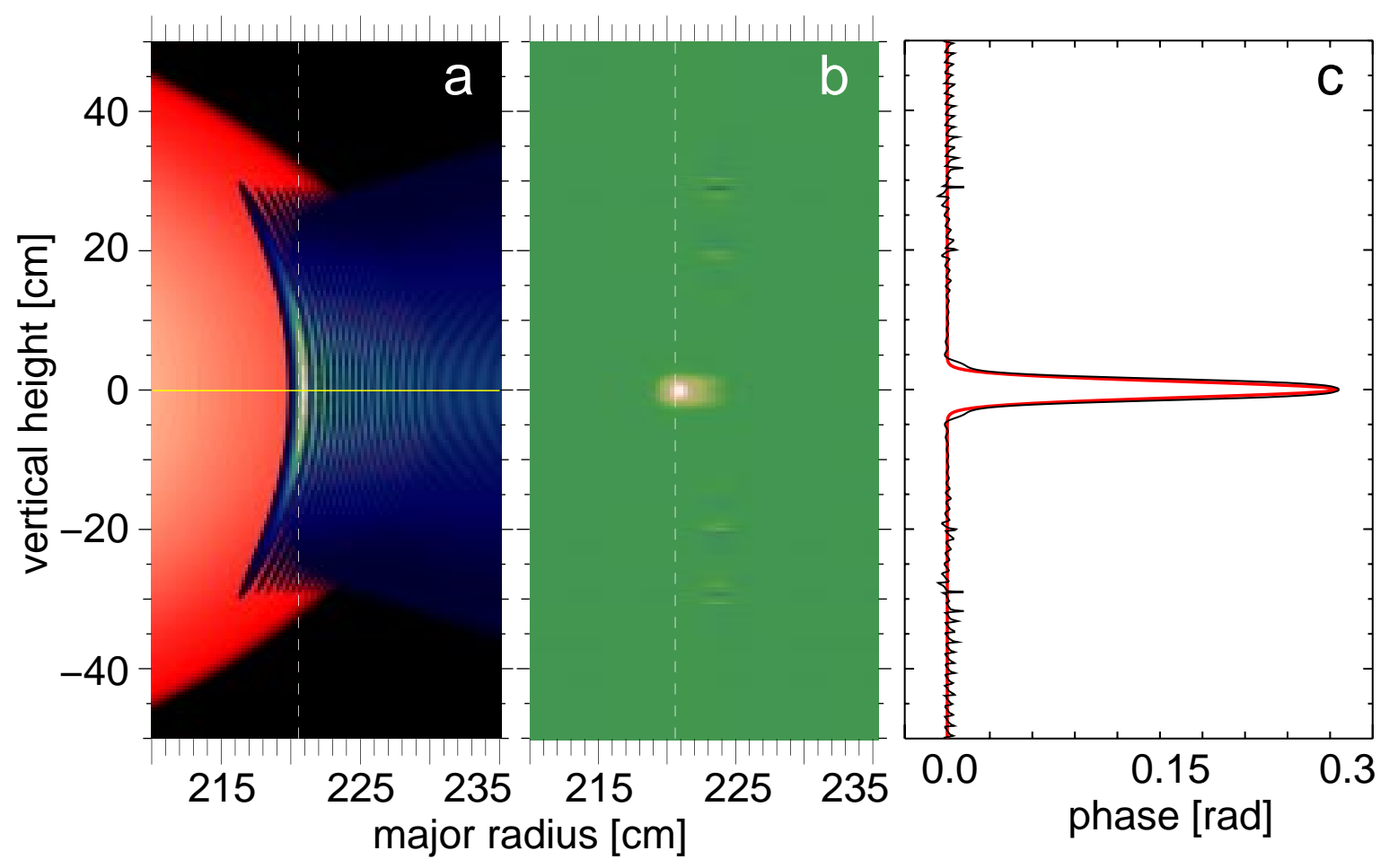

Figure 3. (a) The microwave intensity, $|E|^{2}$, in the vicinity of the cut off layer. The yellow horizontal line indicates the radial and vertical position of a Gaussian density disturbance. The phase at the image plane is shown in (b) as a function of the radial position of the blob. The white dashed line is the location of the cut off layer at the mid plane. The phase response at the image plane (black curve) and the density perturbation (red curve) is shown in (c) for the case where the blob is located at the cut off layer.

$(\tilde{E}=A \exp (i \tilde{\phi}))$, provided that the fluctuation level is small. The minimum in $\tilde{A}$ also decreases with increasing density fluctuation levels.

At the image plane we can indeed use the phase information to form an image of the density perturbation at the cut off so long as the amplitude fluctuations are small as is shown in figure 3 . This was previously demonstrated in slab plane wave geometry in [6]. In this simulation we moved a Gaussian density disturbance with a 1/e width of $1.0 \mathrm{~cm}$ along the optical axis from behind the reflection layer to the edge of the plasma. At the image plane we have recorded the phase change as a function of the position of the poloidal disturbance. In figure $3 \mathrm{~b}$ it is shown that an image is formed of the density disturbance when the blob passes the reflection layer. the change of the phase at the image plane follows accurately the change in the density at the cut off as can be seen in figure $3 \mathrm{c}$. In figure $3 \mathrm{~b}$ a weak response can be seen beyond the cut off layer which can be explained by the fact that the microwaves are sensitive to the density fluctuations along the optical path in the plasma.

In order to investigate the vertical extent of the image plane we have mapped the response at the image plane when we move the Gaussian density disturbance vertically. 


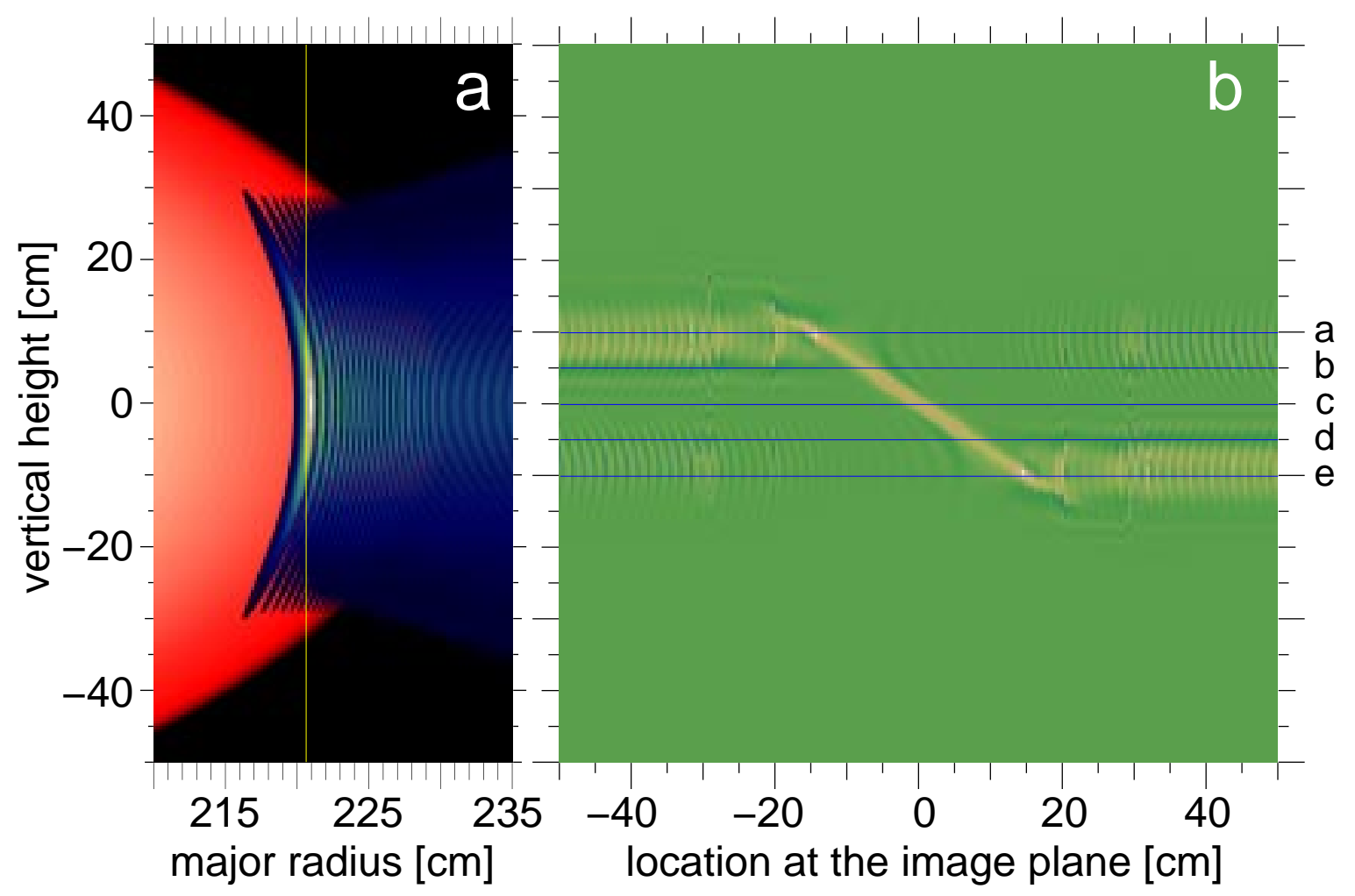

Figure 4. (a) The microwave intensity, $|E|^{2}$, in the vicinity of the cut off layer. The yellow vertical line indicates the radial and vertical position of a Gaussian density disturbance. The phase at the image plane is shown in (b) as a function of the location at the image plane. A clear phase response is obtained where $|E|^{2}$ is high at the cut off layer. The blue lines indicate the location of the cuts shown in figure 5 .

From figure 4 it can be seen that over a range from about $10 \mathrm{~cm}$ below to $10 \mathrm{~cm}$ above the mid plane a reasonable image is formed. Note that this image is 1.5 times enlarged due to the magnification of the optical system. In figure 5 the phase response at the image plane is compared with the density perturbation at the cut off layer. From this figure it can be seen that at the edges of the image plane (frame a at $-15 \mathrm{~cm}$ and $\mathrm{d}$ at $15 \mathrm{~cm}$ ) the images are distorted whereas in between an accurate image of the density perturbation is formed. The decrease in amplitude as shown in figure $5 \mathrm{~b}$ and $\mathrm{d}$ can be explained by plasma curvature effects.

In the imaging simulations so far we have used a lens with an aperture of $100 \mathrm{~cm}$. Decreasing the aperture to $50 \mathrm{~cm}$ degrades the image as can be seen in figure 6: the image plane becomes smaller, it shifts to a larger radius and the amplitude fluctuations increase. It is therefore important for a reflectometer imaging system to use a very large aperture which is often difficult on real machines because of the limited port access. 


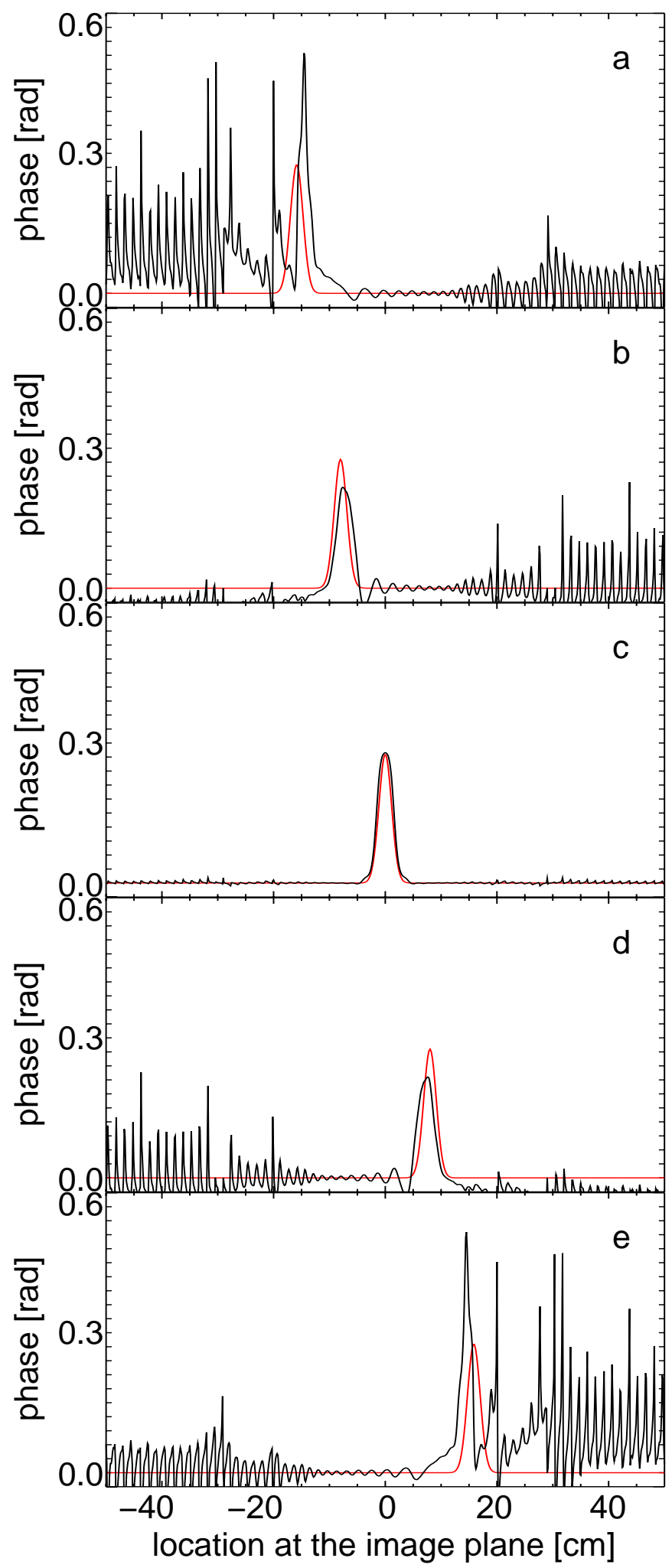

Figure 5. The phase at the image plane (black curve) and the density perturbation (red curve, $\tilde{n} / n=0.1 \%$ ) located at the cut off layer and (a) $10 \mathrm{~cm}$, (b) $5 \mathrm{~cm}$ above, (c) $0 \mathrm{~cm}$ above, (d) $5 \mathrm{~cm}$ below and (e) $10 \mathrm{~cm}$ below the mid plane. 

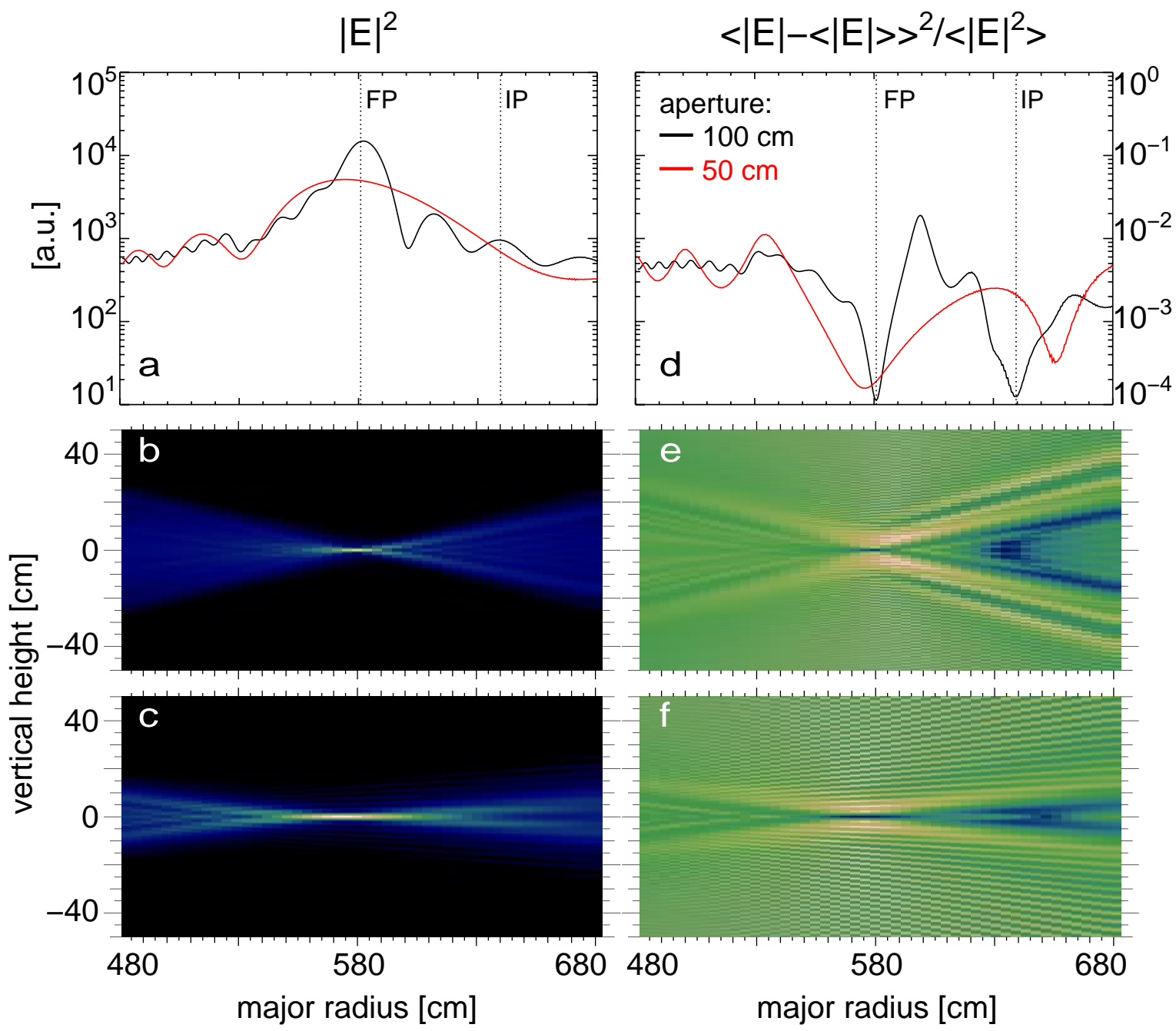

Figure 6. (a) The intensity, $\left\langle|E|^{2}\right\rangle$, of the electrical field along the optical axis for a lens with a diameter of $100 \mathrm{~cm}$ (black) and $50 \mathrm{~cm}$ (red) and $\tilde{n} / n=0.1 \%$. The intensity of the electrical field as a function of major radius and vertical height for a $100 \mathrm{~cm}$ (b) and $50 \mathrm{~cm}$ (c) diameter lens. The intensity increases from black via blue, green, yellow, and pink to white. (d) The relative amplitude fluctuation level along the optical axis for the same two apertures. The amplitude fluctuation level as a function of major radius and vertical height for a lens diameter of $100 \mathrm{~cm}$ (e) and $50 \mathrm{~cm}$ (f). The lines in (e) and (f) outside the region where the beam power is significant are caused by diffraction effects at the lens edges. In (a) and (d) the Fourier plane (FP) and image plane (IM) are indicated with dotted lines.

\section{2. synthetic imaging}

Unlike optical imaging, synthetic imaging mimics the effect of a lens by removing the phase mixing that occurs as a result of propagation away from the cutoff layer. We can form an image of the turbulence in two different ways: as an image in time at a fixed poloidal location or as a poloidal image at a fixed time. In the first approach we use the reflected waves measured with a single detector over a finite time interval. In the second approach the reflected waves measured across a vertical detector array at a 


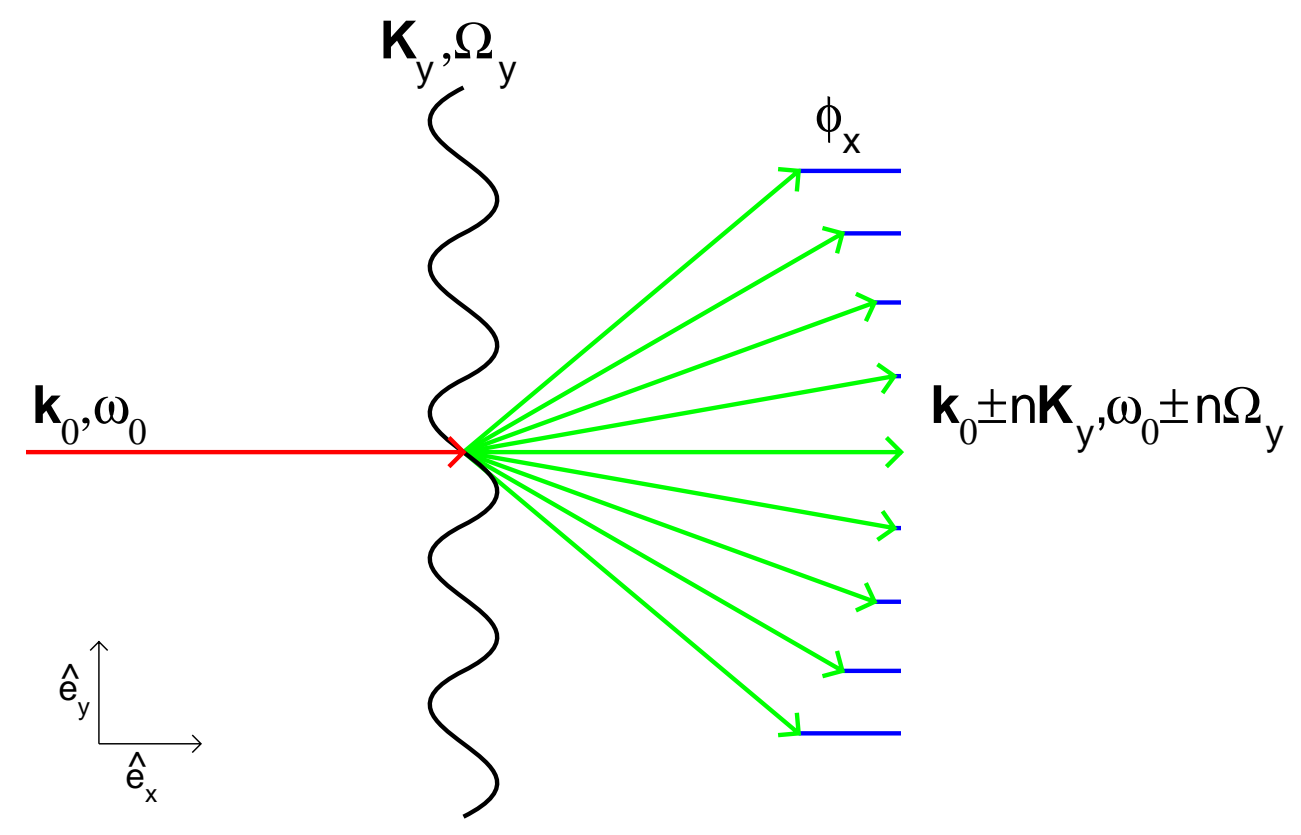

Figure 7. scattering of a microwaves with incident frequency $\omega_{0}$ and wave vector $k_{0}=2 \pi / \lambda_{0}$ from a density perturbation with frequency $\Omega_{y}$ and wave vector $K_{y}$.

single instance are used to reconstruct the density fluctuations at the cut off.

For the first method, using a single detector, consider figure 7 . When a plane wave propagating in the $x$ direction with wave number $k_{0} \hat{e}_{x}$ and frequency $\omega_{0}$ scatters from a density perturbation with a poloidal wave number $K_{y} \hat{e}_{y}$ and frequency $\Omega$, the scattered wave will have a wave number given by $\mathbf{K}_{ \pm}=\left(k_{x}, \pm n K_{y}\right)$ and frequency $\omega_{0} \pm n \Omega$. When $\Omega \ll \omega_{0}$, a condition that is almost always fulfilled in microwave reflectometry, then $k_{x}=\sqrt{k_{0}^{2}-n^{2} K_{y}^{2}} \approx k_{0}-\kappa$ with $\kappa=n^{2} K_{y}^{2} / 2 k_{0}$. It then follows that the phase at a distance $x$ from an object for a given wavenumber $K_{y}$ is $\kappa x$. If the waves propagate with an effective poloidal phase velocity which is directly proportional to the poloidal wave number, as approximately occurs with strongly rotating plasmas, then for each frequency component of the measured signal, $\Omega$, we can apply a phase correction of the form $\exp \left(i \alpha^{2} \Omega^{2} x\right)$ where $x$ is the effective distance from the object and $\alpha$ is the constant of proportionality between the measured frequency and poloidal wavenumber.

Formally the synthesized image, $E(x ; t)$, may be generated by using the following transformation:

$$
E(x ; t)=\mathcal{F}^{-1}\left(\mathcal{F}\left(E\left(x_{0} ; t\right)\right) \mathrm{e}^{\left(i \alpha^{2} \omega^{2}\left(x-x_{0}\right)\right)}\right)
$$

where $\mathcal{F}(\ldots)\left(\mathcal{F}^{-1}(\ldots)\right)$ stands for the Fourier transform (inverse Fourier transform) and $E\left(x_{0} ; t\right)$ is the signal at the detector plane, $x_{0}$, and $x$ is the cut off layer. By varying the back projection parameter $\left(x-x_{0}\right)$ we can identify if there is a virtual location in the plasma where the amplitude fluctuations decrease and the phase fluctuations dominate. This is the plane that formally corresponds to the image plane in a lens based optical system.

In figure 8 there is a clear minimum in the amplitude fluctuations when we vary 


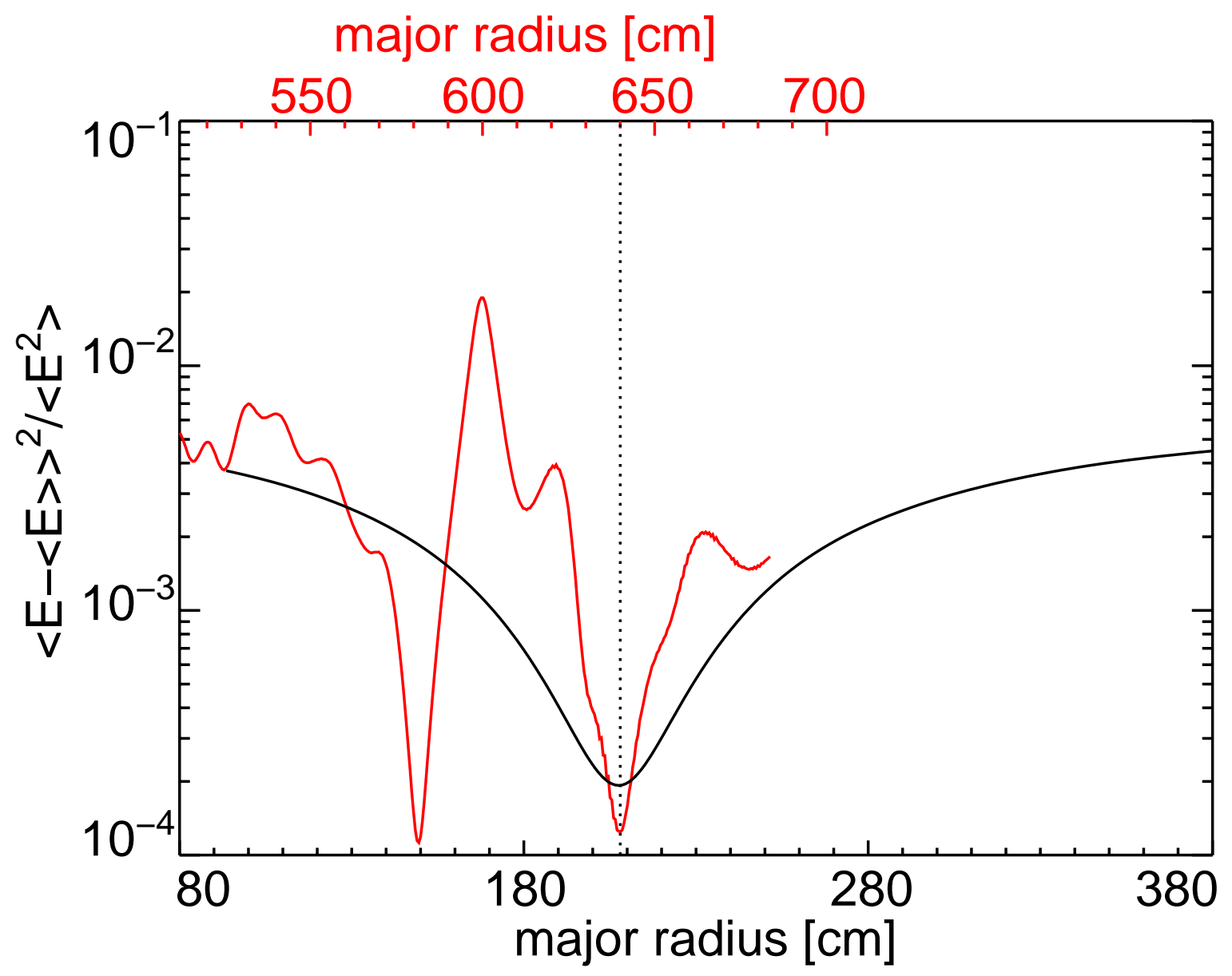

Figure 8. The amplitude fluctuation level for optical imaging (red) and synthetic imaging (black) as a function of the major radius, $R$, with a fluctuation level of $0.1 \%$. The back projection parameter parameter for the synthetic image is $R-3.8$.

the back projection parameter, $\left(x-x_{0}\right)$. The magnitude of this minimum is comparable to the fluctuation minimum at the optical image plane.

As remarked earlier, for synthetic imaging to work well in this approach the fluctuations have to propagate with a single well defined poloidal phase velocity for each $K$. To put it another way, the turbulence can not decorrelate significantly as it propagates across the beam width. The characteristic propagation time of a turbulent eddy across the beam width, defined as $t_{p}=2 /\left(\Delta k_{y} v_{p}\right)$, should be much shorter than the appearent turbulence decorrelation time $\tau$, which means that the eddy has enough time to move past the beam. From this it can be seen that a large $E \times B$ velocity is favorable for this method [12], which can be obtained from neutral beam injection. The characteristic time ratio: $f=\tau / t_{p}$ should be much larger than one for synthetic imaging in time to work properly. This is illustrated in figure 9 where we have used $\tau=0.1 \mathrm{~ms}$ and $1.0 \mathrm{~ms}$ to obtain $f=12.5$ and 125.0 , respectively. The synthetic image at $f=12.5$ is degraded compared to $f=125.0$ i.e. there are significant amplitude fluctuations for $f=12.5$ when the data is projected to the image plane. 


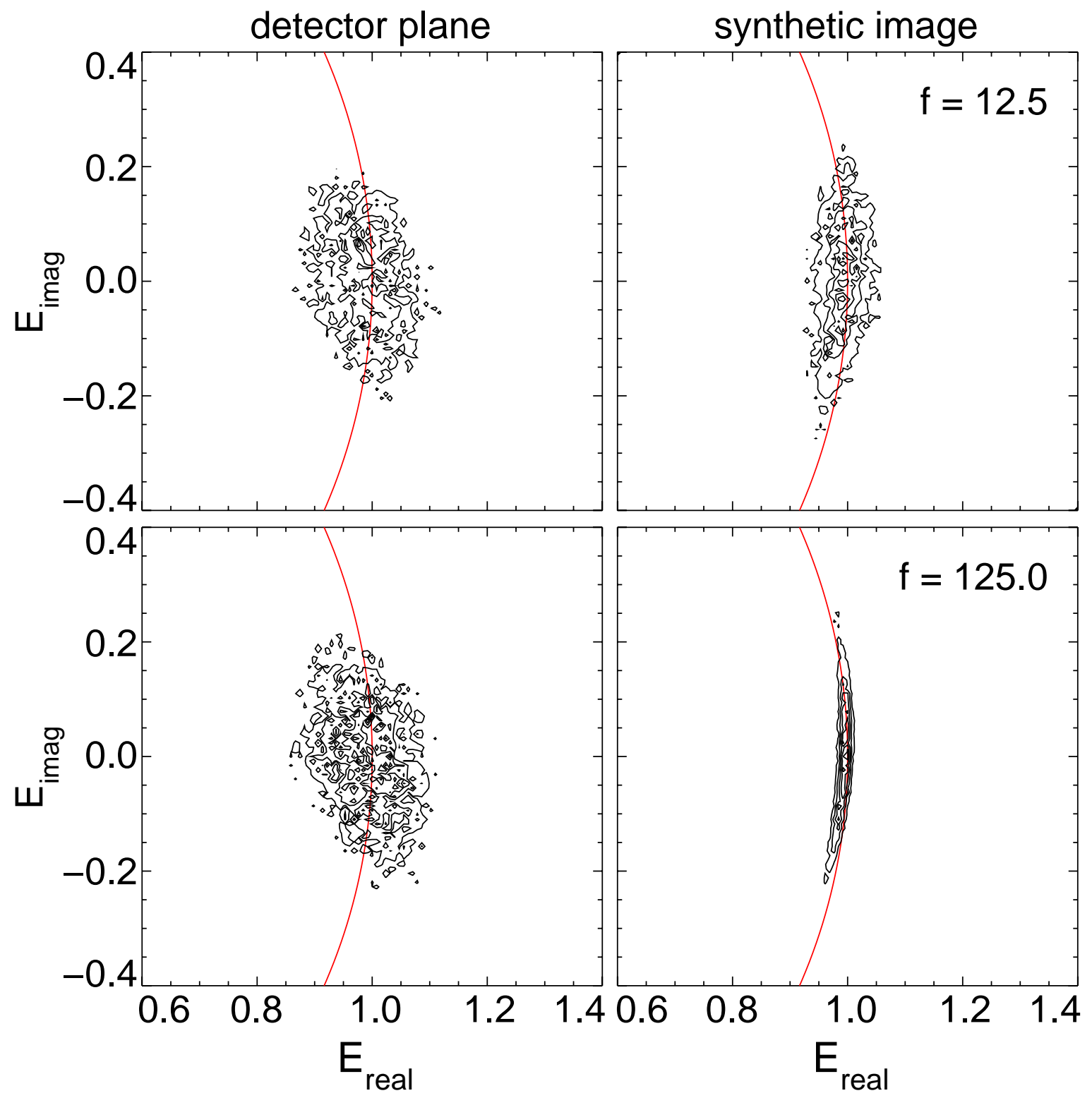

Figure 9. Contour plots of the distribution of the real and imaginary electrical field amplitude. At the detector plane the phase (along the red curve) and amplitude (perpendicular to the red curve) are mixed. Depending on the characteristic time ratio, $f$, we can form an image with a single detector where the amplitude fluctuations are greatly reduced.

To obtain a spatial image of the fluctuations we have to back propagate the scattered electrical field to the effective location of the cut off layer where the amplitude fluctuations are minimal. The electrical field at the cut off layer, $E\left(x_{c}\right)$, is obtained from the electrical field at the detector plane, $E\left(x_{0}\right)$, from:

$$
E\left(x_{c}\right)=\mathcal{F}^{-1}\left(\mathcal{F}\left(E\left(x_{0}\right)\right) \mathrm{e}^{\left(i\left(x_{c}-x_{0}\right) \sqrt{k_{0}^{2}-K_{y}^{2}}\right)}\right)
$$

with $K_{y}$ the perpendicular wave number of the scattered waves. In the next section we 


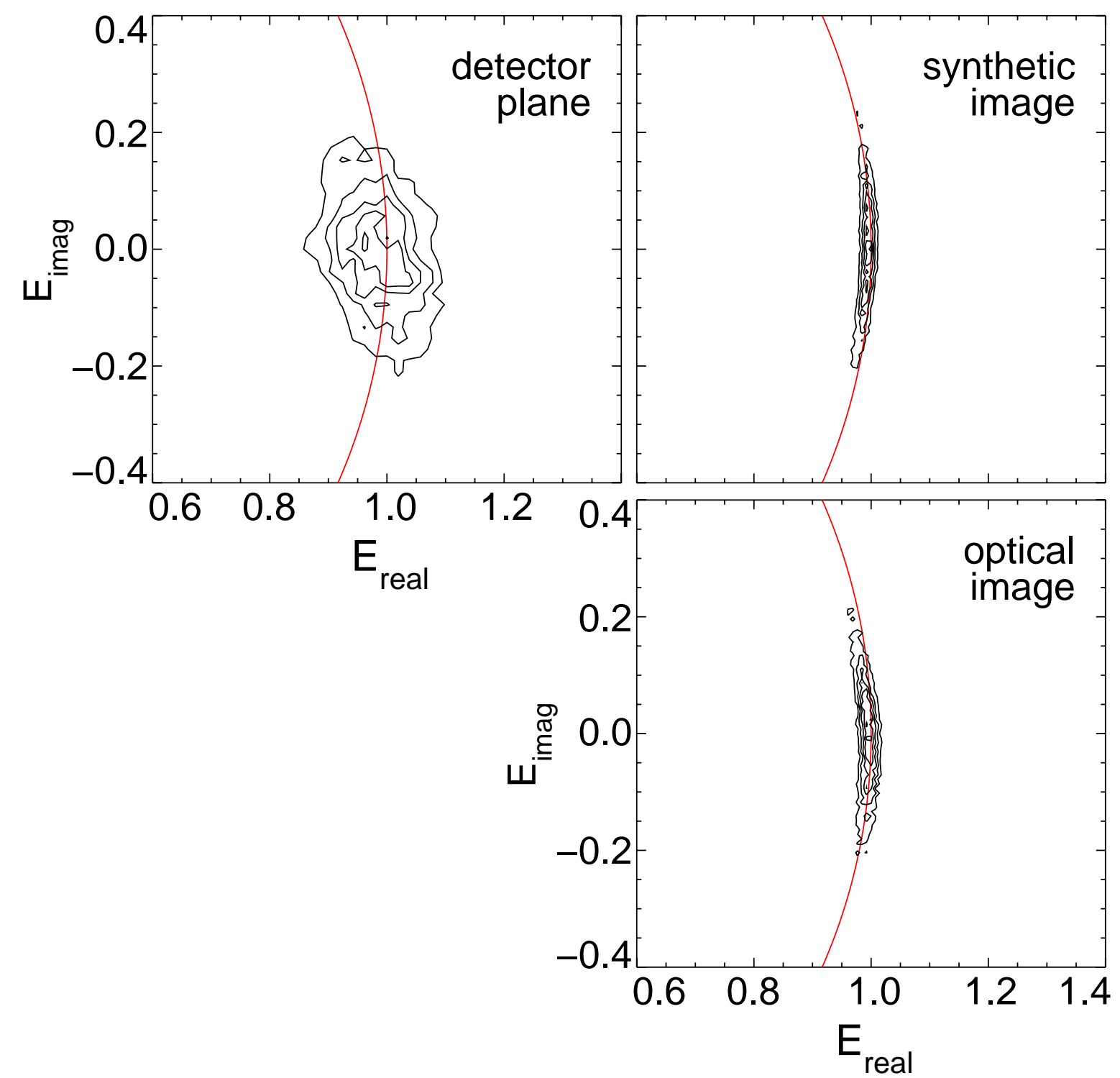

Figure 10. Contour plots of the distribution of the real and imaginary electrical field amplitude. At the detector plane, which coincides with the location of the lens, the phase (along the red curve) and amplitude (perpendicular to the red curve) are mixed. Synthetic and optical imaging reduce the amplitude fluctuations drastically at their respective image planes.

compare both synthetic imaging techniques with the results from optical imaging.

\section{Comparison between optical and synthetic imaging}

\section{1. imaging in time}

We should now be able to reconstruct an image of the density fluctuations at the cut off layer from the (measured) electrical field fluctuations at the synthetic or real image plane. Both optical and synthetic imaging can give the same low relative amplitude 


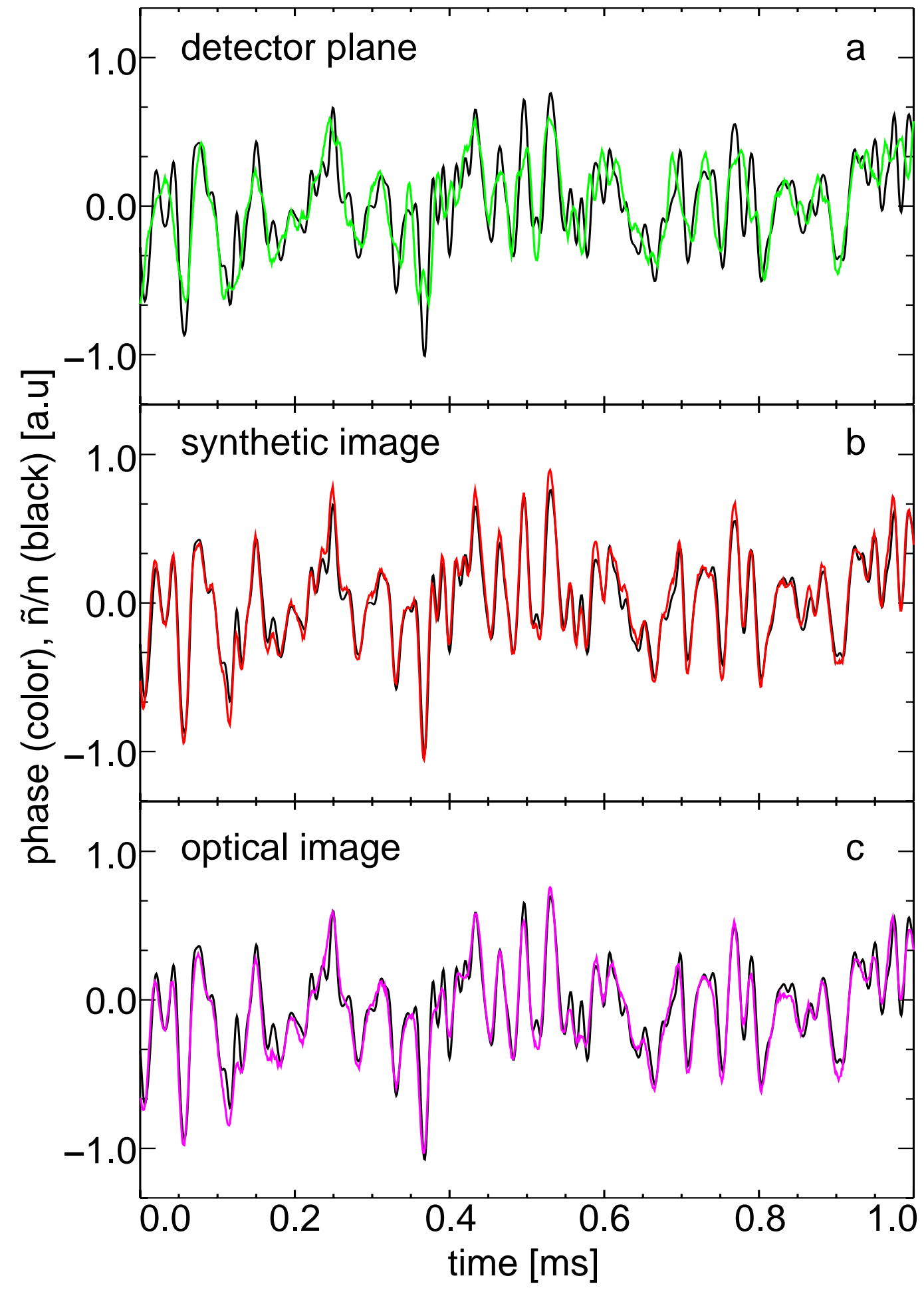

Figure 11. Phase fluctuations (in color) compared to density fluctuations (black) at the cut off layer as a function of time. (a) At the detector plane the phase does not track the density fluctuations accurately. The phase fluctuations after the synthetic (b) and optical (c) imaging follow accurately the density fluctuations at the cut off. In these calculations $k_{r}=0.2 \mathrm{~cm}^{-1}$ was used. 


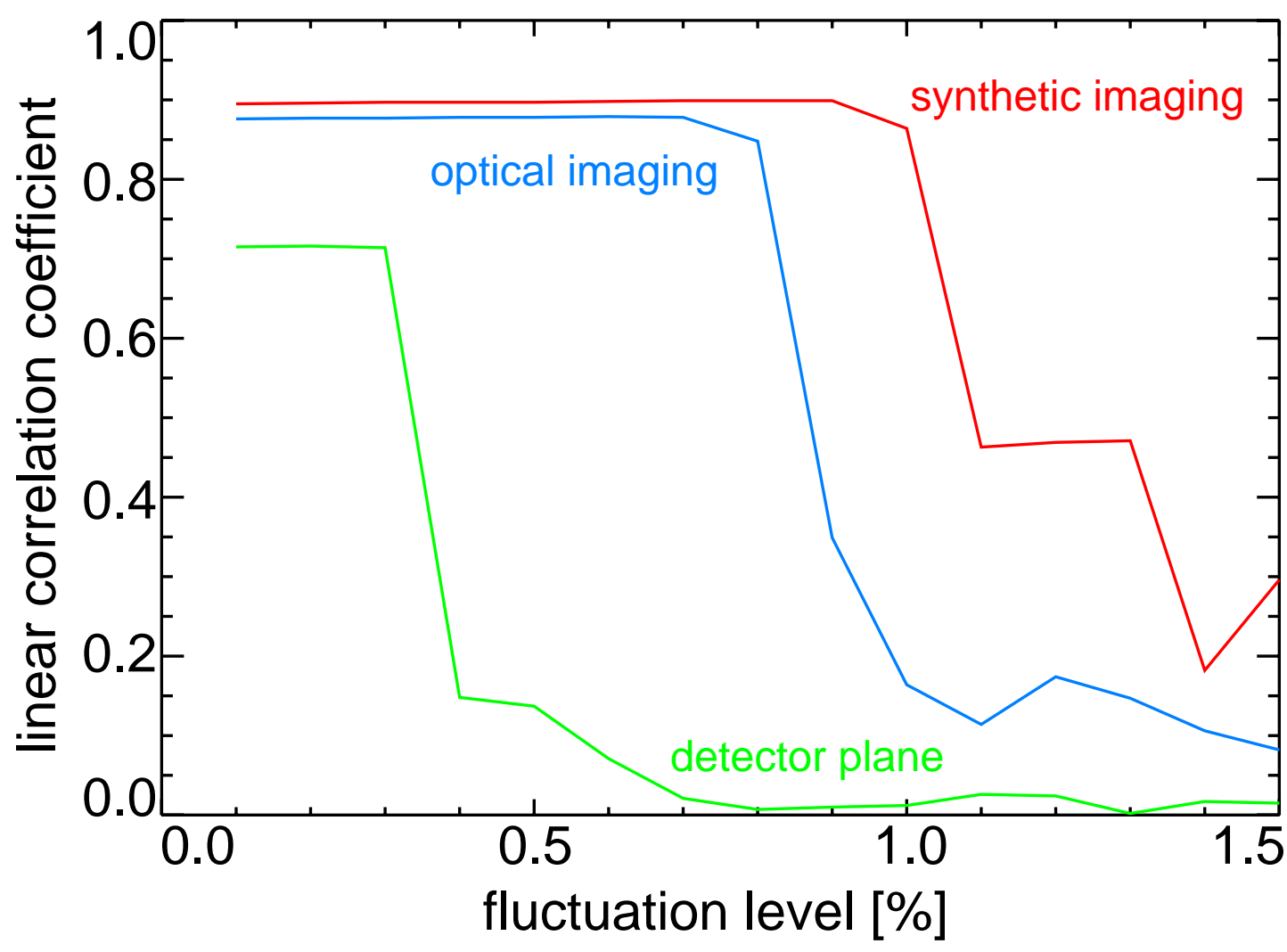

Figure 12. Linear correlation coefficient between the density fluctuations at the cut off layer and the phase fluctuations at the detector plane (green), the image plane of synthetic imaging in time (red), and the image plane of optical imaging (blue) as a function of the fluctuation level.

fluctuations at their respective image planes as is shown in figure 10.

When we compare the phase fluctuations with the density fluctuations at the cut off layer we find that the phase from optical and synthetic imaging follow the density fluctuations at the cut off very well as can be seen in figure 11 .

The results so far were obtained with a density fluctuation level of $0.1 \%$. In most plasmas, however, density fluctuations can be significantly higher. We have investigated the effect of the density fluctuation level by calculating the linear cross correlation coefficient, $r=\langle\tilde{n} \tilde{\phi}\rangle / \sqrt{\left\langle\tilde{n}^{2}\right\rangle\left\langle\tilde{\phi}^{2}\right\rangle}$ with $\tilde{\phi}=\phi-\langle\phi\rangle$, between the density fluctuation at the reflection layer, $\tilde{n}$ and the phase at the optical and synthetic image planes, $\phi$. In figure 12 it is shown that optical imaging works well up to a certain fluctuation level, in our DIII-D equilibrium case up to $\tilde{n} / n=0.8 \%$. Numerical imaging starts to degrade from $\tilde{n} / n=1.0 \%$, indicating a similar limit for detection, provided that $f>100$.

For optical imaging in the microwave range of frequencies it is important to keep in mind that there may be significant diffraction effects due to the finite aperture of the receiving lens. These diffraction effects from the edges of aperture can contaminate the image plane (see figure 6) and may limit optical imaging. The reason for this contamination is that the returning beam is typically spread over much larger angles 


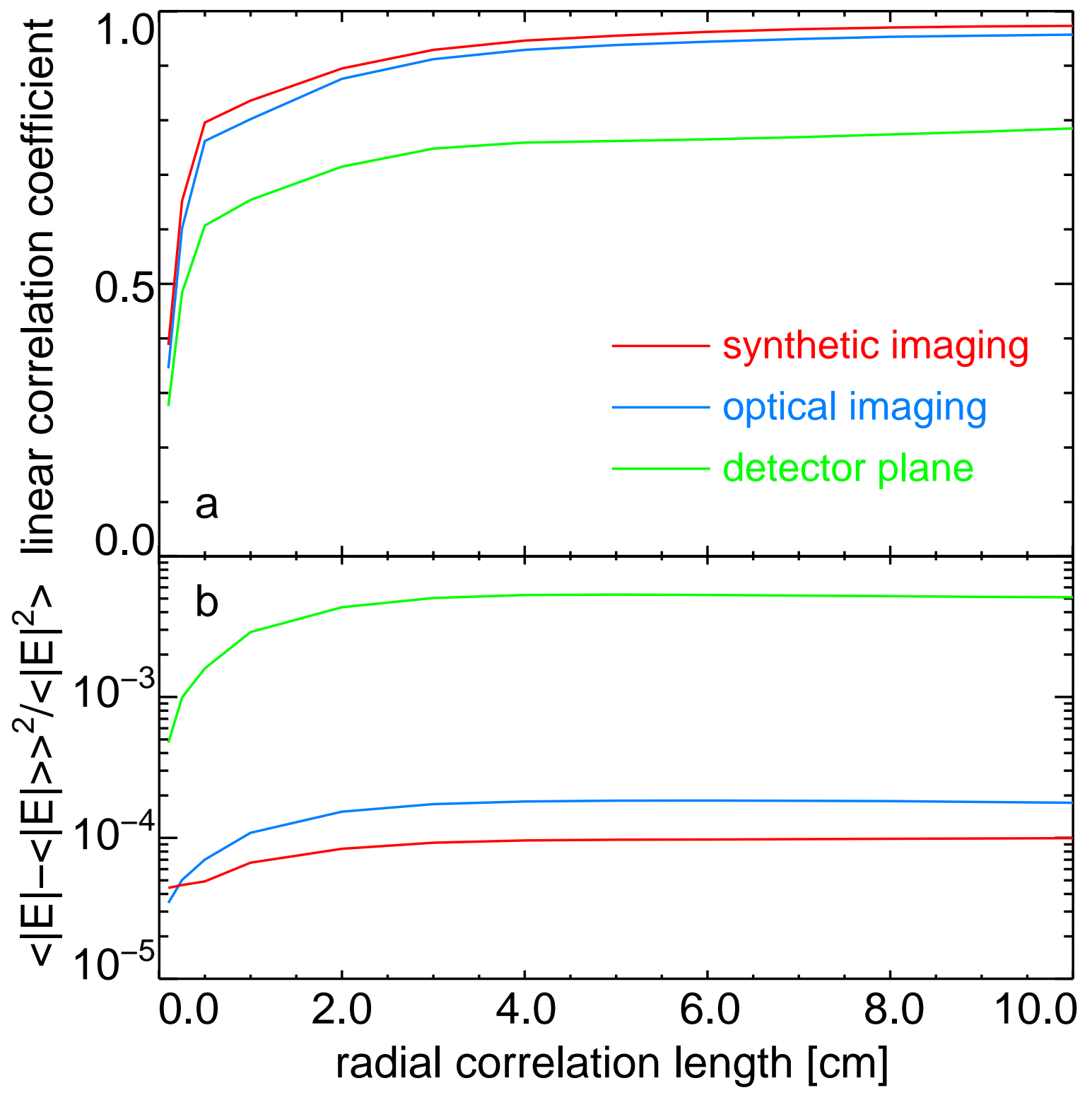

Figure 13. (a) Linear correlation coefficient between the density fluctuations at the cut off layer and the phase fluctuations at the detector plane (green), the image plane of synthetic imaging in time (red), and the image plane of optical imaging (blue) as a function of the radial correlation length, $\lambda=2 / k_{r}$. At short radial correlation lengths the synthetic and optical imaging degrade. (b) Relative amplitude fluctuations as a function of the radial correlation length for synthetic (red) and optical (blue) imaging and at the detector plane (green).

than can be collected by an optical element. This spread is mostly due to the curvature of the plasma. Numerical back projection is mostly immune to those diffraction effects.

Reflectometry is mainly sensitive to the density fluctuations close to the reflection layer, however, fluctuations along the whole ray path can affect the measurement. In order to investigate this effect we have varied the radial correlation length, $\lambda=2 / k_{r}$, between 0.1 and $10.0 \mathrm{~cm}$ and calculated the linear cross correlation coefficient, $r$, 
between the density fluctuation at the reflection layer and the phase at the optical and synthetic image planes. As the radial correlation length increases ( $k_{r}$ decreases) the cross correlation increases for both the optical and synthetic imaging (see figure 13a). Moreover, the correlation coefficients for synthetic and optical imaging are very much the same. At short radial correlation lengths (large $k_{r}$ ) the correlation starts to decrease for both methods, which is expected due to the short radial wavelengths. This decrease is not correlated with an increase of the relative amplitude fluctuations at the image planes as is shown in figure 13b. In fact the relative amplitude fluctuations are also decreasing when the correlation between the fluctuations at the cut off and its image is decreasing. Therefore, knowledge of the radial correlation length is needed to determine if imaging is performed with either an optical or synthetic imaging system.

\section{2. imaging in space}

For the synthetic imaging using a vertical detector array we have taken the electrical field at the detector plane of one simulation and projected it back to the reflection layer. In contrast to the imaging in time case of the previous section where we have taken into accout the effects of the finite size of the receiving antenna, we have not taken into account these here for the detector array. By using the electrical field at the dector plane we obtain an upper limit of the imaging possibilities. The effects due to a finite detector size are beyond the scope of this paper. At fluctuation levels up to $1 \%$ we get good image quality for both the optical and synthetic imaging over the poloidal range that is lit by the microwave beam as is shown in figure 14a. At a higher fluctuation level of $3 \%$, however, the optical and synthetic imaging both fail (figure 14b).

In order to exploit the limits for optical imaging and synthetic imaging with a detector array to the density fluctuation level and radial wave number, we have generated time series from the imaged data at the optical axis for all the samples in each simulation and calculated the linear correlation coefficient between the density fluctuations at the cut off layer and the phase fluctuations of the imaged signals. We have scanned the radial correlation length (figure 15) at a fixed density fluctuation level of $0.1 \%$ and the density fluctuation level (figure 16) at a fixed radial wave number of $1.0 \mathrm{~cm}^{-1}$.

From figure 15 it can be seen that both optical and synthetic imaging in space behave similarly with respect to the radial density correlation length. For high $\lambda$ (low $k_{r}$ ) the correlation is high whereas at low $\lambda$ (high $k_{r}$ ) the correlation decreases rapidly.

For low fluctuation levels optical and synthetic imaging in space are equivalent but the optical imaging technique starts to fail at lower fluctuation levels than the synthetic imaging (see figure 16) although the difference is not significant.

Unlike synthetic imaging in time, which is restricted to poloidally rotating plasmas, synthetic imaging using a vertical detector array has no such restriction. 


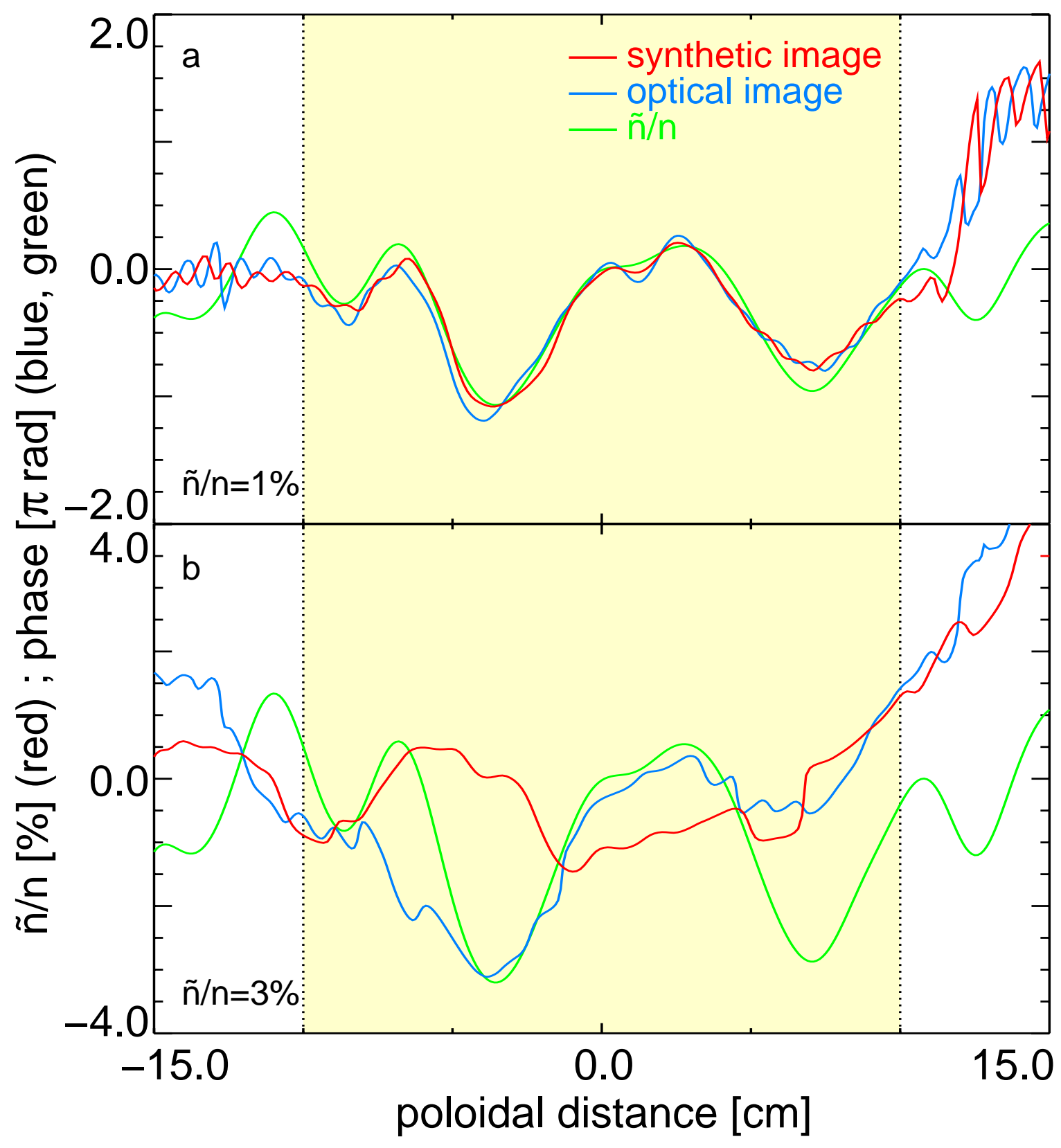

Figure 14. Phase fluctuations from synthetic in space (red) and optical imaging (blue) compared to density fluctuations (green) at the cut off layer as a function of poloidal distance for a fluctuation level of $1 \%$ (a) and $3 \%$ (b). The yellow region between $\pm 10 \mathrm{~cm}$ was lit by the incoming microwave beam. At a $1 \%$ fluctuation level a good image is formed with both optical and synthetic imaging techniques whereas at $3 \%$ both techniques fail. 


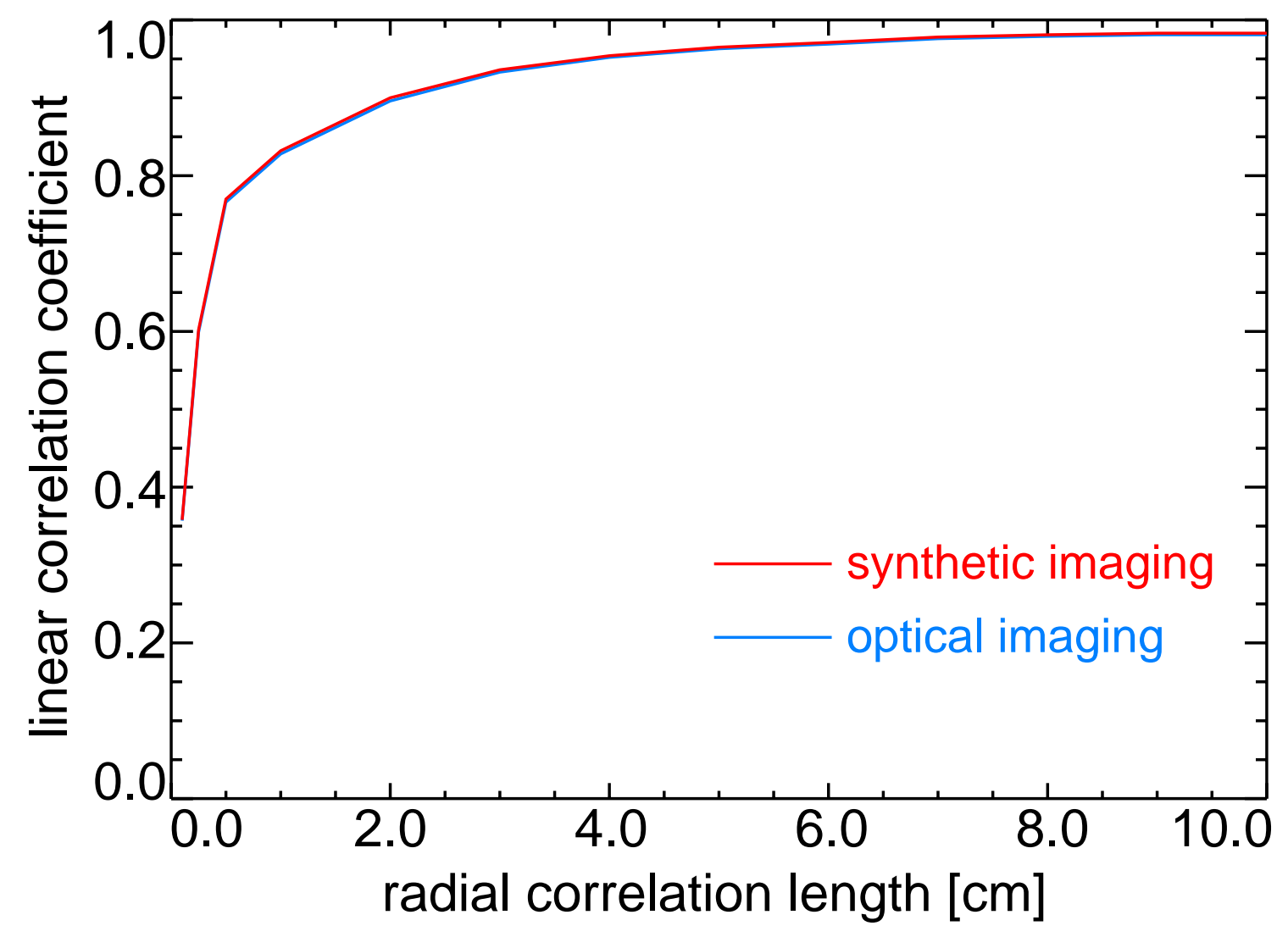

Figure 15. Linear correlation coefficient between the density fluctuations at the cut off layer and the phase fluctuations at the image plane of synthetic imaging in space (red) and the image plane of optical imaging (blue) as a function of the radial correlation length, $\lambda=2 / k_{r}$. At short radial correlation lengths the synthetic and optical imaging degrade.

\section{Discussion and future directions}

In the previous sections we explored the quality of imaging that can be obtained with reflectometry using synthetic and optical imaging methods. One of the most striking results of this study is the extent to which synthetic imaging methods may be employed to reconstruct density fluctuations without complex optical systems. The synthetic imaging methods also have advantages of independently imaging multiple frequency beams.

Numerical back projection in time can already be performed with existing heterodyne reflectometer systems provided that turbulent eddies propagate poloidally as if frozen into the plasma. If this condition is not well satisfied then the quality of the single detector synthetic image degrades and methods based on detector arrays will be superior. This hypothesis of frozen-in turbulence can be tested in experiments where two receivers are placed in the poloidal direction. From a time delay correlation analysis between the two signals the vertical drift velocity and the correlation decay length in the frame of the drifting turbulence can be independently determined. Such a study 


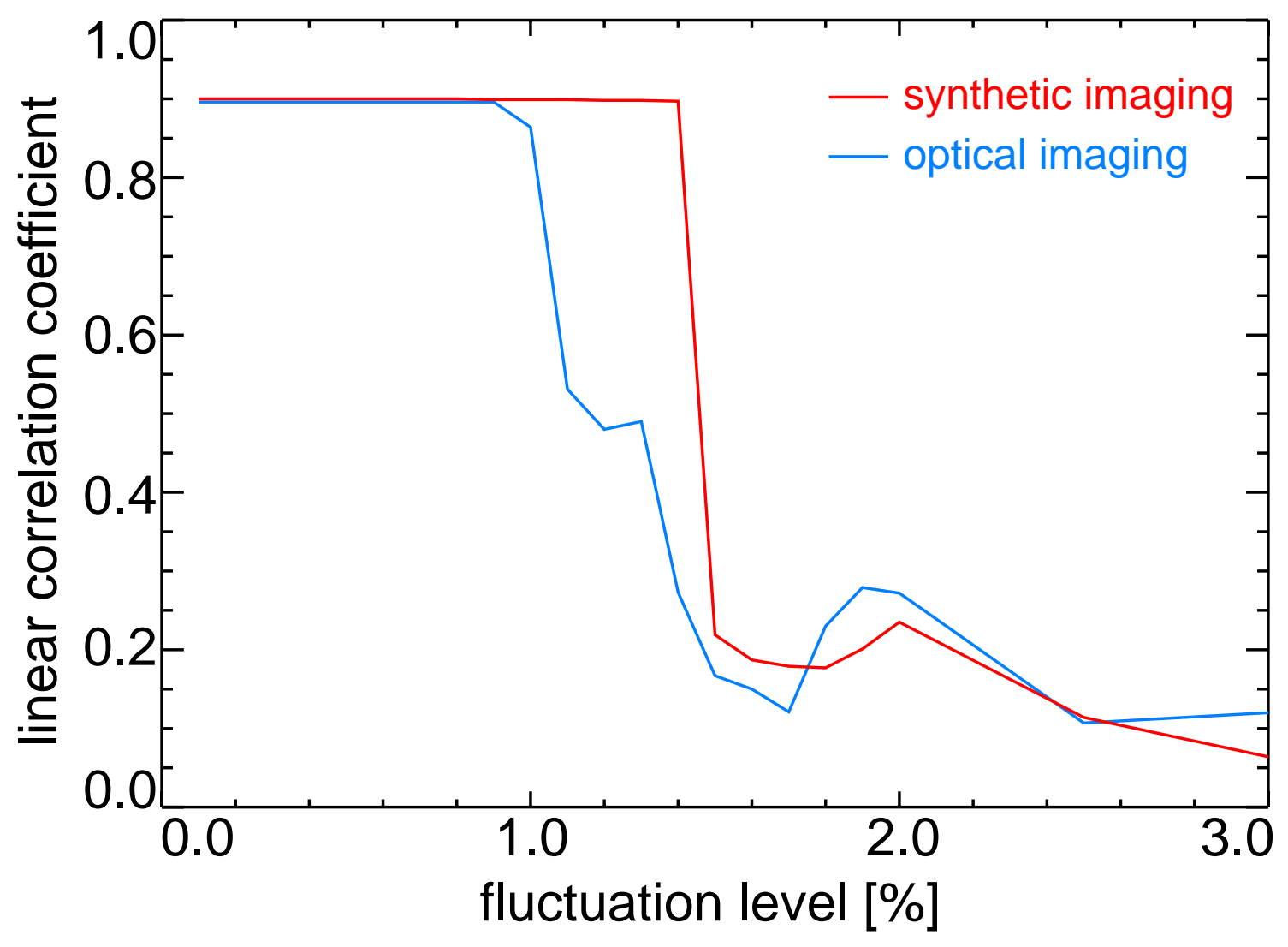

Figure 16. Linear correlation coefficient between the density fluctuations at the cut off layer and the phase fluctuations at the image plane of synthetic imaging in space (red), and the image plane of optical imaging (blue) as a function of the fluctuation level.

using two point correlation methods has already been used to ascertain the $\mathrm{E} \times \mathrm{B}$ flow of turbulent eddies [12].

Numerical back projection in space requires the construction of a poloidal array of receivers. On current machines where the port access may be too limited to incorporate a large lens an internal array may be an attractive option. The signals from this array can then be used for synthetic imaging in space as explained above (section 4.2).

When addressing the issue of synthetic versus optical imaging in the microwave range of frequency it is important to keep in mind two very critical issues: i) the effect of the finite aperture of the receiving lens and ii) the signal resolution of the detector. One limitation of optical imaging is the effect of diffraction from apertures, which can contaminate the image, as seen in figure 6 . In the case of synthetic imaging the effect of edge discontinuities can be greatly reduced. The only requirement is that the receivers measure the fluctuating electric field with high fidelity. However, with the use of modern receivers and high power sources the fidelity of the signal can easily exceed $30 \mathrm{~dB}$ which should be adequate for imaging.

So long as the radial correlation length of the turbulence is significantly longer 
than the beam wave length, the phase at the image plane corresponds closely to the density structures at the cutoff layer. This is heartening, given that the beam is passing through multiple structures on its way in and out of the plasma. However, as the radial correlation length decreases, there is a degradation of the image quality even when the amplitude fluctuations are weak at the image plane. The reason for this is that the phase is now better represented as an ensemble average over independent turbulent structures and over multiple radial correlation lengths in the path of the beam. Thus knowledge of the radial scale length of density fluctuations is essential to determine if imaging is being performed. In practice an imaging system, optical or synthetic, that claims to measure local density fluctuations must combine the imaged phase with the measurement of the radial correlation length of the fluctuations.

\section{Acknowledgments}

This work was supported by DOE Contract No. DE-AC02-76-CH03073. 
microwave reflectometry Imaging

\section{References}

[1] Nazikian R and Mazzucato E, 1995 Rev. Sci. Instrum. 66392

[2] Mazzucato E, 1998 Rev. Sci. Instrum. 691691

[3] Nazikian R 1997 Journal of Modern Optics 441037

[4] Valeo E, Kramer G J, and Nazikian R 2002 Plasma Phys. Control. Fusion 44 L1

[5] Conway G D 1997 Plasma Phys. Control. Fusion 39407

[6] Mazzucato E 2001 Nucl. Fusion 41203 and references therein

[7] Lin Y, Nazikian R, Irby J H and Marmar E S 2001 Plasma Phys. Control. Fusion 43 L1

[8] Donne A J H, Jaspers R, Barth C J, Bindslev H, Elzendoorn B S Q, van Gorkom J C, van der Meiden H J, Oyevaar T, van der Pol M J, Udintsev V S, Widdershoven H L M, Biel W, Finken K H, Krämer-Flecken A, Kreter A, Oosterbeek H, Schweer B, Untenberg B, Deng B H, Domier C W, Luhmann Jr N C, Mazzucato E, Munsat T, Park H, Porte L, Woskov P and Shmaenok L 2001 Rev. Sci. Instrum. 721046

[9] Munsat T, Mazzucato E, Park H, Deng B H, Domier C W, Luhmann Jr. N C, Wang J, Xia Z G, Donné A H J and van de Pol M, 2003 Rev. Sci. Instrum. 741426

[10] Munsat T, Mazzucato E, Park H, C W Domier C W, N C Luhmann Jr N C, Donné A H J and M van de Pol M, 2003 Plasma Phys. Control. Fusion 45469

[11] Nazikian R, Kramer G J, and Valeo E 2001 Phys. Plasmas 81840

[12] Carlstrom T N, Groebner R J, Fenzi C, McKee G R, Moyer R A, and Rhodes T L 2002 Plasma Phys. Control. Fusion 44 A333 


\section{External Distribution}

Plasma Research Laboratory, Australian National University, Australia

Professor I.R. Jones, Flinders University, Australia

Professor João Canalle, Instituto de Fisica DEQ/IF - UERJ, Brazil

Mr. Gerson O. Ludwig, Instituto Nacional de Pesquisas, Brazil

Dr. P.H. Sakanaka, Instituto Fisica, Brazil

The Librarian, Culham Laboratory, England

Mrs. S.A. Hutchinson, JET Library, England

Professor M.N. Bussac, Ecole Polytechnique, France

Librarian, Max-Planck-Institut für Plasmaphysik, Germany

Jolan Moldvai, Reports Library, Hungarian Academy of Sciences, Central Research Institute for Physics, Hungary

Dr. P. Kaw, Institute for Plasma Research, India

Ms. P.J. Pathak, Librarian, Institute for Plasma Research, India

Ms. Clelia De Palo, Associazione EURATOM-ENEA, Italy

Dr. G. Grosso, Instituto di Fisica del Plasma, Italy

Librarian, Naka Fusion Research Establishment, JAERI, Japan

Library, Laboratory for Complex Energy Processes, Institute for Advanced Study, Kyoto University, Japan

Research Information Center, National Institute for Fusion Science, Japan

Dr. O. Mitarai, Kyushu Tokai University, Japan

Dr. Jiangang Li, Institute of Plasma Physics, Chinese Academy of Sciences, People's Republic of China

Professor Yuping Huo, School of Physical Science and Technology, People's Republic of China

Library, Academia Sinica, Institute of Plasma Physics, People's Republic of China

Librarian, Institute of Physics, Chinese Academy of Sciences, People's Republic of China

Dr. S. Mirnov, TRINITI, Troitsk, Russian Federation, Russia

Dr. V.S. Strelkov, Kurchatov Institute, Russian Federation, Russia

Professor Peter Lukac, Katedra Fyziky Plazmy MFF UK, Mlynska dolina F-2, Komenskeho Univerzita, SK-842 15 Bratislava, Slovakia

Dr. G.S. Lee, Korea Basic Science Institute, South Korea

Institute for Plasma Research, University of Maryland, USA

Librarian, Fusion Energy Division, Oak Ridge National Laboratory, USA

Librarian, Institute of Fusion Studies, University of Texas, USA

Librarian, Magnetic Fusion Program, Lawrence Livermore National Laboratory, USA

Library, General Atomics, USA

Plasma Physics Group, Fusion Energy Research Program, University of California at San Diego, USA

Plasma Physics Library, Columbia University, USA

Alkesh Punjabi, Center for Fusion Research and Training, Hampton University, USA

Dr. W.M. Stacey, Fusion Research Center, Georgia Institute of Technology, USA

Dr. John Willis, U.S. Department of Energy, Office of Fusion Energy Sciences, USA

Mr. Paul H. Wright, Indianapolis, Indiana, USA 
The Princeton Plasma Physics Laboratory is operated by Princeton University under contract with the U.S. Department of Energy.

\author{
Information Services \\ Princeton Plasma Physics Laboratory \\ P.O. Box 451 \\ Princeton, NJ 08543
}

Phone: 609-243-2750

Fax: 609-243-2751

e-mail: pppl_info@pppl.gov

Internet Address: http://www.pppl.gov 